\title{
A RELAÇÃO ENTRE INFORMAÇÃO, CULTURA ORGANIZACIONAL E TOMADA DE DECISÃO EM UMA ORGANIZAÇÃO
}

\author{
LA RELACIÓN ENTRE LA INFORMACION, \\ CULTURA ORGANIZACIONAL Y TOMA DE \\ DECISIONES EN UNA ORGANIZACIÓN
}

\author{
Paulo Danelon Lopes - paulodanelon@yahoo.com.br \\ Doutorando em Ciência da Informação pela Universidade de \\ Brasília (UnB). \\ Valmira Perucchi - vperucchi2@yahoo.com.br \\ Doutora em Ciência da Informação pela Universidade de Brasília. \\ (UnB). Bibliotecária do Instituto Federal de Educação, Ciência e \\ Tecnologia da Paraíba (IFPB).
}

\section{RESUMO}

Introdução: Compreende uma pesquisa documental sobre a relação entre informação, cultura organizacional e tomada de decisão em uma organização. Objetivo: verificar qual a influência da informação, considerando-se a cultura organizacional, na tomada de decisão em uma organização.

Metodologia: A revisão de literatura procura contemplar autores especializados nas áreas de informação (Belkin; Borko; Capurro; Choo; Tarapanoff; dentre outros); cultura (Fleury et al.; Moraes e Fadel; Nassar e Schein); tomada de decisão (Angeloni; Hoppen; Leitão e Nassif; Lousada e Valentim e Oliveira) e organização (Bernardes e Marcondes e Maximiano). Resultados: pode existir uma grande interdependência entre informação, cultura e tomada de decisão em uma organização.

Conclusões: A informação pode facilitar a compreensão da cultura de uma organização, de como ocorrem os processos de mudança e quais alternativas podem ser levantadas para que ela possa lograr êxito em seu processo de tomada de decisão com o objetivo de assegurar a sua perpetuação ao longo do tempo.

Palavras-chave: Informação para organização, cultura no ambiente organizacional, tomada de decisão em organização, informação no processo decisório. 


\section{INTRODUÇÃO}

O cotidiano de qualquer ser humano é estar inserido em inúmeras organizações ao longo de toda a sua existência, uma vez que é por intermédio dessas que ele pode conseguir a sua realização pessoal e profissional nos campos político, econômico, psicossocial, científico e militar e assegurar a sobrevivência de sua espécie.

Segundo Lapassade (1972), a noção de organização pode apresentar dois significados distintos: coletividades (fábricas, sindicatos, bancos e associações diversas) que buscam objetivos, tais como produção de bens ou a sua distribuição; a formação de homens ou a gestão de seus lazeres e condutas sociais e certos processos sociais que procuram organizar a mobilização dos meios para atingir os objetivos coletivos (produzir, educar, distribuir) de uma comunidade.

Um dos grandes objetivos de qualquer organização é a sua perpetuação ao longo do tempo. No entanto, tal propósito não é fácil de ser alcançado, pois inúmeros obstáculos surgem ao longo de sua existência. Uma das formas pela qual a organização pode atingir a perenidade é por intermédio de um adequado processo de uso da informação. A coleta de dados e a transformação desses dados em informações necessárias ao processo decisório de uma organização nos níveis operacional, tático e estratégico pode auxiliar na consecução desse objetivo.

A cultura se faz presente em qualquer organização, independente do ramo de atuação da empresa, do seu nicho de mercado, do país em que atua e do nível intelectual, econômico e social de seus trabalhadores em todos os níveis hierárquicos. Para Hofstede (1991), cultura é uma programação coletiva da mente que distingue os membros de um grupo ou categoria de pessoas face a outro. Nos manuscritos de Shein (2009), cultura é um fenônemo dinâmico que nos cerca em todas as horas, sendo constantemente desempenhada e criada por nossas interações com outros e moldada por comportamento de liderança e um 
Paulo Danelon Lopes; Valmira Perucchi

A relação entre informação, cultura organizacional e tomada de decisão em uma organização

conjunto de estruturas, rotinas, regras e normas que orientam e restringem o comportamento.

Pode-se afirmar que a informação, bem como a busca de dados, a gestão do conhecimento, o desenvolvimento da inteligência organizacional e o processo decisório fazem parte da rotina diária de uma organização.

O objetivo desta revisão de literatura é, sem esgotar o assunto, procurar apresentar a relação que pode existir entre informação, cultura organizacional e o processo de decisório de uma organização que esteja focada na manutenção de sua sobrevivência e aperfeiçoamento de um produto ou serviço para a sociedade. Essa revisão pretende contribuir para ampliar a compreensão do tema nas áreas de Ciência da Informação e Administração.

A natureza desta revisão de literatura, em conformidade com a classificação proposta por Gil (2010) com base no seu objetivo, é do tipo descritiva, apresentando características de identificação e determinação dos construtos Informação, Cultura Organizacional e Tomada de Decisão e Organização em relação à influência da informação na transformação da cultura organizacional que busca o aperfeiçoamento do processo decisório. Quanto ao procedimento, a presente pesquisa pode ser classificada como pesquisa bibliográfica e documental.

Além desta introdução, o presente artigo apresenta mais oito seções, a primeira aborda o que é informação, a segunda o que é cultura organizacional, a terceira o processo de tomada de decisão em uma organização, a quarta o que é organização, a quinta trata da relação entre informação e organização, a sexta avalia a influência da cultura no ambiente organizacional, a sétima é sobre a ingerência da informação no processo de tomada de decisão e a oitava são as considerações finais. 


\section{O QUE É INFORMAÇÃO?}

Ao se buscar definir o que é informação é fundamental o estudo dos Fundamentos da Ciência da Informação, uma vez que essa disciplina procura tratar dos inúmeros conceitos de informação sob o ponto de vista de diversos autores focados no estudo dessa área.

Para Borko (1968), a Ciência da Informação é uma ciência interdisciplinar que procura investigar as propriedades e comportamentos da informação, as forças que direcionam o fluxo e uso da informação $e$ as técnicas do processamento da informação necessárias ao armazenamento, recuperação e disseminação. Esse autor levou em consideração as três definições de ciência da informação de Taylor: o fato de a ciência da informação ser uma disciplina que investiga as propriedades e comportamento da informação, as forças que governam o fluxo da informação e os meios de processamento da informação para acessibilidade e usabilidade.

Para Hoskovsky e Massey (apud WERSIG; NEVELING, 1975): "a informação é um processo que ocorre dentro da mente humana quando são colocados numa união produtiva um problema e os dados úteis para sua solução."

De acordo com Belkin (1978), um conceito adequado para informação deve levar em consideração as necessidades do usuário da informação, pois usuários diferentes podem responder ao mesmo conjunto de dados de forma diferente, em momentos distintos e em função da maneira como os dados Ihe são disponibilizados para uso.

Para Capurro (2003), um sistema de informação deve estar preparado para propiciar o suporte adequado à produção, coleta, organização, interpretação, armazenamento, recuperação, disseminação, transformação e uso de conhecimentos. Sendo assim, ele deve ser concebido no marco de um grupo social concreto e para áreas específicas. 
De acordo com Capurro e Hjorland (2007), a informação não se restringe aos fatos, pois depende do contexto e das limitações impostas. Ela é um paradigma social e não individual. Ela é conceitual e cultural.

Leitão e Nassif (2009, p. 138) estabelecem uma relação entre informação, decisão estratégica e sensemaking organizacional. Os autores tentam incluir uma nova abordagem para pesquisas que investigam a relação entre o uso da informação e a tomada de decisões estratégicas pela organização, partindo do pressuposto de que "o valor da informação para a tomada de decisão não está na quantidade de informação e sim no significado que pode ser extraído desta relação para construir o ambiente organizacional e orientar uma ação." (LEITÃO; NASSIF, 2009, p. 138). Ainda, com relação à informação Leitão e Nassif (2009, p, 137), consideram "uma alternativa para ampliar a nossa observação sobre informação e decisões estratégicas poderia ser um modelo que considerasse a informação como recurso para a construção coletiva de significado em uma organização."

Pode-se dizer que o conteúdo da informação a ser disponibilizado para a análise e solução de um problema, dentro de um contexto específico, deva levar em consideração as necessidades e particularidades daqueles que a utilizam. Em razão de a interpretação da informação poder variar de acordo com o contexto, estudar-se-á cultura organizacional.

\section{O QUE É CULTURA ORGANIZACIONAL?}

Para verificar a interdependência entre informação e tomada de decisão em uma organização é importante compreender o que é cultura organizacional e a sua influência nessa relação.

De acordo com Schein (2009), ao longo da história da humanidade, o conceito de cultura tem sido utilizado de inúmeras formas: pelos advogados como uma palavra para indicar sofisticação, quando se diz que alguém é muito culto; pelos antropólogos para referir- 
Paulo Danelon Lopes; Valmira Perucchi

A relação entre informação, cultura organizacional e tomada de decisão em uma organização

se aos costumes e rituais que as sociedades desenvolvem no curso de sua história; por alguns pesquisadores organizacionais e gerentes, com o intuito de se referir ao clima e às práticas que as organizações desenvolvem ao lidar com pessoas, ou aos valores expostos e ao credo de uma organização.

Cultura, normalmente, contribui para certo nível de estabilidade estrutural em um grupo. Ao se dizer que algo é cultural, pode-se afirmar que não é apenas compartilhado pelos integrantes de uma comunidade, mas também estável, uma vez que ajuda a definir as inúmeras características de uma sociedade.

Ao abordar cultura organizacional, Nassar (2000) registra que ela é um conjunto de valores, crenças e tecnologias que pode contribuir para a união dos mais diferentes membros de uma organização em diversos níveis hierárquicos na superação de obstáculos em busca da consecução de objetivos organizacionais.

Na análise de Schein (apud FLEURY et al.,1996, p. 20):

[...] cultura organizacional é o conjunto de pressupostos básicos que um grupo inventou, descobriu ou desenvolveu ao aprender como lidar com os problemas de adaptação externa e integração interna e que funcionaram bem o suficiente para serem considerados válidos e ensinados a novos membros como a forma correta de perceber, pensar e sentir, em relação a esses problemas.

Moraes e Fadel (2007) investigam e analisam as relações entre cultura e comportamento organizacional diante da adoção de novas tecnologias no ambiente informacional de uma organização. Partem do pressuposto que uma maneira viável de readequar uma organização é rever a sua Cultura. Dessa forma, "Estudar a cultura organizacional em cenários de mudança é fundamental para entender os processos que envolvem as organizações". As Organizações cada vez mais se dão conta que a informação é um recurso estratégico que tem custo, preço e valor. Desta forma, existe a necessidade dela ser gerenciada assim como os recursos financeiros, materiais e humanos. 
Verifica-se que a cultura de uma organização é dinâmica em função das necessidades de uma organização estar sempre se adaptando ao cenário ao seu redor para se perpetuar ao longo do tempo e atender as necessidades da sociedade. Diante da perspectiva da influência da cultura em uma organização, discorrer-se-á sobre o processo de tomada de decisão.

\section{O PROCESSO DE TOMADA DE DECISÃO EM UMA ORGANIZAÇÃO}

Considerando-se que todas as organizações são sistemas, isto é, um conjunto de partes que se encontram em constante interação e interdependência na execução de inúmeras funções para a consecução de um ou mais objetivos em prol da sociedade, o processo de tomada de decisão faz parte do seu cotidiano, uma vez que é por intermédio dele que as ações a serem desenvolvidas são decididas.

Hoppen (1992) percebe a organização como um sistema em constante mudança e acredita que as atividades das empresas em todos os seus níveis hierárquicos são essencialmente atividades de tomada de decisão e de resolução de problemas.

Para Oliveira (2004, p.143): "o processo de tomada de decisão implica uma racionalidade objetiva que traz como consequência a necessidade de o tomador de decisão ajustar seu comportamento a um sistema integrado, por meio de uma visão ampla de alternativas que se Ihe afiguram antes da tomada de decisão."

De acordo com Angeloni (2003), a tomada de decisão em uma organização tem exigido cada vez mais o trabalho em equipe. $\mathrm{Na}$ interação entre esses grupos deve existir um processo de comunicação que estimule o diálogo.

Para Lousada e Valentim (2011, p. 148), a tomada de decisão é uma temática relevante na atualidade, "uma vez que as organizações 
Paulo Danelon Lopes; Valmira Perucchi

A relação entre informação, cultura organizacional e tomada de decisão em uma organização

necessitam, cada vez mais, de decisões acertadas, em um espaço de tempo mais reduzido."

A tomada de decisão é inerente ao trabalho de qualquer pessoa, Lousada e Valentim (2011, p. 163), compreendem que no "caso dos gestores, a tomada de decisão faz parte de sua função, ou seja, é tão importante quanto qualquer outra atividade sob sua responsabilidade."

Leitão e Nassif (2009, p. 150), concluem que a "informação mantém uma relação intrínseca com o processo decisório na medida em que é utilizada com o objetivo de auxiliar a compreender a situação e indicar um caminho a seguir."

Constata-se que o processo de tomada de decisão faz parte da rotina de qualquer organização nos seus diversos níveis, mas o que seria uma organização em sua essência?

\section{O QUE É ORGANIZAÇÃO?}

De acordo com Maximiano (2009, p. 3): "uma organização é um sistema de trabalho que transforma recursos em produtos e serviços." Os recursos envolvidos nessa transformação podem ser humanos, materiais, financeiros, informação, espaço e tempo.

Para Bernardes e Marcondes (2009, p.14): "a organização é uma coletividade formada por pessoas que tem a função de produzir bens e prestar serviços à sociedade, bem como atender às necessidades de seus participantes. Ela possui uma estrutura formada por indivíduos que se relacionam colaborando e dividindo o trabalho."

Nesse contexto, questiona-se, há relação entre informação e organização? 


\section{A RELAÇÃO ENTRE INFORMAÇÃO E ORGANIZAÇÃO}

Uma das grandes revoluções pela qual o mundo passou foi a Revolução Científica, quando o ser humano sentiu a necessidade de buscar parâmetros para explicar os fenômenos da natureza. Outro grande passo da humanidade foi a Revolução Industrial, onde se buscou amplificar o poder de produção. Atualmente, o mundo vive a Revolução da Informação, consequência da necessidade de amplificar o poder do pensamento da sociedade para o levantamento de alternativas e tomadas de decisão que assegurem a escolha das melhores ações a serem implementadas para a preservação da espécie humana e uma melhor qualidade de vida.

Segundo Resende (2006), a informação é todo o dado trabalhado, útil, tratado, com valor significativo atribuído ou agregado a ele e com um sentido natural e lógico para quem usa a informação. $O$ dado é entendido como um elemento da informação, um conjunto de letras, números ou dígitos, que, tomado isoladamente, não transmite nenhum conhecimento.

Para Choo (2006), a busca e o processamento da informação são fundamentais em muitos sistemas sociais e atividades humanas, pois as necessidades e usos da informação devem ser examinados dentro de um contexto profissional, organizacional e social dos usuários. Talvez essa seja a razão pela qual o uso da informação venha se tornando um componente cada vez mais importante da pesquisa nas áreas de recuperação da informação, sistemas de informação e tomada de decisão dentre outras.

Os usuários obtêm informações de muitas e diferentes fontes formais e informais. As fontes informais, inclusive colegas e contatos pessoais, são quase sempre tão ou mais importante que as fontes formais, como bibliotecas e banco de dados on-line.

Um grande número de critérios pode influenciar a seleção e o uso das fontes de informação. As pesquisas descobriram que muitos grupos 
Paulo Danelon Lopes; Valmira Perucchi

A relação entre informação, cultura organizacional e tomada de decisão em uma organização

de usuários preferem fontes locais e acessíveis, que não são, necessariamente, as melhores. Para esses usuários, a acessibilidade de uma fonte de informação é mais importante que sua qualidade.

De acordo com Choo (2006, p. 97):

[...] os executivos, por exemplo, não empreendem buscas abrangentes nem procuram as melhores soluções. Ao contrário, buscam a informação localmente, usando fontes conhecidas e quase sempre procurando soluções na vizinhança dos problemas. Os problemas são considerados resolvidos quando uma solução suficientemente boa é encontrada.

Para Davenport (1998), há quatro modalidades de informações dentro das organizações: a) informações não estruturadas existentes dentro das organizações, mas, normalmente, não relacionadas a um processo formal de trabalho ou relacionadas, mas não tratadas pelo processo; b) capital intelectual ou conhecimento - abrangendo as competências individuais, as competências organizacionais e a retenção e disseminação do conhecimento; c) informações estruturadas em papel - como registros pessoais e arquivo-morto que evidenciam a importância da consciência do profissional em tratar adequadamente seus registros, atuando mesmo como um gerente de registros, tomando as medidas necessárias para o adequado armazenamento e disseminação do que foi importante e útil para a empresa e d) informações estruturadas em computadores que normalmente refletem o ambiente informacional das organizações.

Pode-se concluir, com base nos pressupostos acima que: a informação faz-se presente em todas as etapas da vida humana, pois subsidia o processo decisório individualmente e dentro dos grupos sociais; suas fontes podem ser formais e informais e moldadas de forma estrutural dentro da organização, por intermédio do capital intelectual ou conhecimento e estruturadas em papel ou computadores.

Consciente da relação entre informação e organização é importante que se analise a influência da cultura no ambiente 
organizacional, pois cada organização tem seus valores, normas e regras específicas de convivência e trabalho.

\section{$\begin{array}{llllll}7 & \text { A INFLUENNCIA } & \text { DA } & \text { CULTURA NO } & \text { AMBIENTE }\end{array}$ ORGANIZACIONAL}

Se a cultura de um grupo é o resultado de sua aprendizagem acumulada, como descrever e catalogar o conteúdo dessa aprendizagem? Quaisquer grupos e teorias organizacionais distinguem dois importantes conjuntos de problemas com que todos os grupos, não importam seus tamanhos, devem lidar: (1) sobrevivência, crescimento e adaptação em seu ambiente e (2) integração interna, que permite o funcionamento diário e a capacidade de adaptar-se e aprender (SCHEIN, 2009).

Para Hofstade (1991), a cultura é adquirida, não herdada. Ela provém do ambiente social do indivíduo, não dos genes. O estudo da cultura revela que os grupos humanos e categorias pensam, sentem e agem de forma diferente, mas não existem parâmetros científicos que permitam considerar um grupo intrinsecamente superior ou inferior a outro. É necessária a reflexão antes de se aplicar as normas de um indivíduo, grupo ou sociedade, a outros. O julgamento e a ação devem ser precedidos de informação sobre a natureza das diferenças culturais entre sociedades, suas raízes e conseqüências.

De acordo com Davenport (1998), o comportamento informacional é uma dimensão inexplorada e vasta do gerenciamento informacional, qualquer ação de gestão da informação que não considera as pessoas como o fator determinante de sucesso está fadada ao fracasso.

Sob o ponto de vista de Choo (1998), por meio da cultura informacional, forma-se uma lógica que dirige a atenção dos membros da organização. Desta forma, alguns dados são considerados, outros ignorados e incorporados à estratégia, aos sistemas, aos valores e as expectativas dos membros da organização. Grande parte dos aspectos 
relacionados à cultura informacional acaba se transformando em regras e rotinas que determinam formas para levar a cabo a coleta, a distribuição e o uso das informações.

Em harmonia com as ideias delineadas de pesquisadores registradas nos parágrafos anteriores, conclui-se de forma embrionária que a cultura pode influenciar a estabilidade de um grupo; ela é adquirida e não herdada; que antes de se aplicar normas a um indivíduo, grupo ou sociedade deve-se buscar informação sobre a natureza das diferenças culturais e que a cultura informacional pode interferir no ambiente organizacional.

\section{A INGERÊNCIA DA INFORMAÇÃO NO PROCESSO DE TOMADA DE DECISÃO DE UMA ORGANIZAÇÃO}

Segundo Wilson (1997), a gestão da informação pode ser definida como a aplicação de princípios administrativos à aquisição, organização, controle, disseminação e uso da informação para a operacionalização efetiva de organizações de todos os tipos.

Para Froehlich (1989), os cientistas, ou profissionais da informação podem ser considerados mediadores, educadores e facilitadores do processo de acesso e disseminação da informação, o que provoca mudanças na ordem social.

Segundo Tarapanoff (2006) o principal objetivo da gestão da informação é identificar e potencializar recursos informacionais de uma organização ou empresa e sua capacidade de informação, ensinando-a a aprender e a se adaptar a mudanças ambientais.

A informação é um fator determinante para a melhoria de processos, produtos e serviços, tendo valor estratégico em organizações. A ideia da informação como ferramenta estratégica evoluiu depois que a gestão da informação mudou seu foco inicial de gestão de documentos e dados para recursos informacionais, mostrando 
Paulo Danelon Lopes; Valmira Perucchi

A relação entre informação, cultura organizacional e tomada de decisão em uma organização

resultados em relação à eficiência operacional, evitando desperdício e automatizando processos.

De acordo com Wilson (1997), gestão da informação preocupa-se com o valor, qualidade, posse, uso e segurança da informação no contexto do desempenho organizacional.

De acordo com Mcgee e Prusak (1994), a estratégia é definida a partir da informação, avaliando as oportunidades ou ameaças existentes e a sua capacidade de acionar os seus ativos para responder aos novos desafios, tem sido a ocupação principal dos líderes e tomadores de decisão.

Para Lev (2004), com a intensificação do uso da informação para fins estratégicos, percebeu-se que o valor da empresa, na percepção do usuário e do mercado, incorpora aspectos "intangíveis", tais como valor da marca, peso das patentes geradas, capacidade de inovação, talento dos funcionários em especial dos executivos e suas relações com os clientes, software, processos únicos, desenhos organizacionais e outros.

Segundo Tarapanoff (2006, p. 28): "as organizações devem usar a informação para criar significado, construir conhecimento e tomar decisões. A criação do conhecimento representa inovação e vantagem competitiva."

Após análise das ideias transcritas acima, as seguintes conclusões parciais podem ser alcançadas: todos aqueles que são gestores da informação podem influenciar no processo de mudança social; a informação pode ser crucial para a análise e melhoria de processos, produtos e serviços em uma organização e também contribuir para a criação de significados, construção de conhecimento, tomada de decisões, inovação e vantagem competitiva no que concerne a decisões antecipadas e ações pro ativas. 


\section{CONSIDERAÇÕES FINAIS}

A informação está presente em todo ambiente organizacional. A sua correta gestão pode ser peça fundamental ao processo manutenção e crescimento de uma organização dentro do cenário na qual ela está inserida, uma vez que ela: compreende o resultado da coleta e tratamento de dados, contribui para o aumento do conhecimento organizacional, monitora a cultura organizacional e está sempre presente nos processos de tomada de decisão.

A utilização de informações que atendam aos princípios da oportunidade e exatidão pode contribuir para a melhoria de processos, produtos e serviços, tendo valor estratégico em organizações que buscam uma existência duradoura. Tal afirmativa está em consonância com Leitão e Nassif (2009), pois não importa a quantidade de informação, mas sim, o significado da informação para orientar ações e construir significado ao ambiente organizacional.

A análise da cultura de um determinado grupo pressupõe a busca de informações sobre as características dessa célula da sociedade, pois a cultura não é herdada mais adquirida por intermédio de uma reflexão.

A cultura informacional (conjunto de valores, atitudes $e$ comportamentos que influenciam a forma como a pessoa avalia, aprende, recolhe, organiza, processa, comunica e utiliza a informação) é capaz de propiciar considerável subsídio a organizações que vivenciam um constante processo de mudança necessário ao cumprimento de sua missão e sobrevivência no mercado.

Pode-se concluir de acordo com Angeloni (2003); Belkin (1978); Bernardes e Marcondes (2009); Borko (1968); Capurro (2003); Choo (1998, 2006); Fleury et al. (1996); Hoppen (1992); Leitão e Nassif (2009); Lousada e Valentim (2011); Maximiano (2009); Moraes e Fadel (2007); Nassar (2000); Oliveira (2004); Schein (2009) e Tarapanoff (2006) que há uma grande interdependência entre informação, cultura organizacional e tomada de decisão em uma organização. Por meio da 
Paulo Danelon Lopes; Valmira Perucchi

A relação entre informação, cultura organizacional e tomada de decisão em uma organização

informação pode-se compreender a cultura de uma organização, como ocorrem os processos de mudança e quais alternativas podem ser levantadas para que o processo de tomada de decisão seja conduzido.

A informação deve procurar atender às necessidades particulares de seus usuários, uma vez que esses irão utilizá-la em um contexto cultural para tomarem decisões eficientes, eficazes e efetivas dentro das organizações em que estão inseridos.

Para buscar a elucidação das premissas abordadas ao longo desse trabalho, ratificando-as ou retificando-as, seria interessante a realização de pesquisas de campo em organizações com o propósito de se verificar a interdependência entre informação, cultura e tomada de decisão; como os colaboradores de uma organização utilizam a informação; como as organizações lidam com a cultura; como é a adaptação das organizações aos novos cenários, levando em consideração a informação e a cultura organizacional para a tomada de decisão e verificar como ocorre na prática a relação entre informação e a tomada de decisão.

\section{REFERÊNCIAS}

ANGELONI, Maria Terezinha. Elementos intervenientes na tomada de decisão. Ciência da Informação, Brasília, v. 32, n. 1, p. 17-22, jan./abr. 2003.

BELKIN, Nicholas J. Information concepts for information science. Journal of Documentation, London, v. 34, n. 1, p. 55-85, mar. 1978.

BERNARDES, Cyro; MARCONDES, Reynaldo C. Teoria geral da administração: gerenciando as organizações. 3. ed. São Paulo: Saraiva, 2009.

BORKO, H. Information systems technology staff system development corp. Santa Monica, Califórnia. American Documentation, Washington, v. 19, n. 1, p.3-5, Jan. 1968. 
CAPURRO, Rafael. Epistemologia e ciência da informação. In:

ENCONTRO NACIONAL DE PESQUISA EM CIÊNCIA DA

INFORMAÇÃO, 5., Belo Horizonte, 2003. Anais .... Belo Horizonte, 2003. Disponível em: <http://www.capurro.de/enancib_p.htm>. Acesso em: 22 set. 2012.

CAPURRO, Rafael; HJORLAND, Berger. O conceito de informação. Tradução de Ana Maria P. Cardoso, Maria da Glória A. Ferreira e Marco Antônio de Azevedo. Perspectiva em Ciência da Informação, Belo Horizonte, v. 12, n. 1, p. 148-207, jan./abr. 2007.

$\mathrm{CHOO}$, Chun Wei. Information management for the intelligent organization: the art of scanning the environment. $2^{\text {nd }}$ ed. Medford, $\mathrm{NJ}$ : American Society for Information Science by Information Today, 1998.

CHOO, Chun Wei. A organização do conhecimento: como as organizações usam a informação para criar significado, construir conhecimento e tomar decisões. 2. ed. São Paulo: Ed. SENAC, 2006.

DAVENPORT, Thomas. Ecologia da informação: porque só a tecnologia não basta para o sucesso na era da informação. São Paulo: Futura. 1998.

FLEURY, Maria Tereza Leme et al. Cultura e poder nas organizações. São Paulo: Atlas, 1996.

FROEHLICH, T. J. The foundations of information in social epistemology. In: ANNUAL HAWAII INTERNATIONAL CONFERENCE ON SYSTEM SCIENCES, 22., 1989, Washington. Proceedings ... Washington, D.C.: IEF Computer Science Press, 1989. p. 306-315.

GIL, Antonio Carlos. Como elaborar projetos de pesquisa. 5. ed. São Paulo: Atlas, 2010.

HOFSTEDE, Geert H. Culturas e organizações: compreender a nossa programação mental. Lisboa: Sílabo, 1991.

HOPPEN, Norberto. Resolução de problemas, tomada de decisões e sistemas de informações. Caderno de Administração Geral, Porto Alegre, set. 1992.

LAPASSADE, Georges; LOURAU, René. Chaves da sociologia. Rio de Janeiro: Civilização Brasileira, 1972. 
LEITÃO, Pedro Claudio Coutinho; NASSIF, Mônica E. Decisões estratégicas e informação: sensemaking organizacional como abordagem alternativa. Estratégia e Negócios, Florianópolis, v. 2, n. 1, p. 136-157. jan./jun. 2009. Disponível em:

$<$ http://portaldeperiodicos.unisul.br/index.php/EeN/index>. Acesso em: 4 jun. 2013.

LEV, Baruch. Sharpening the intangibles edge. Harvard Business Review, Boston, p. 108-116, jun., 2004.

LOUSADA, Mariana; VALENTIM, Marta Lígia Pomim. Modelos de tomada de decisão e sua relação com a informação orgânica. Perspectivas em Ciência da Informação, Belo Horizonte, v. 16, n. 1, p. 147-164, jan./mar. 2011.

MAXIMIANO, Antonio Cesar Amaru. Fundamentos de administração. 2. ed. São Paulo: Atlas, 2009.

MCGEE, James; PRUSAK, Laurence. Gerenciamento estratégico da informação. 10. ed. Rio de Janeiro: Campus, 1994.

MORAES, Cássia R. B.; FADEL, Bárbara. Cultura organizacional em cenários de mudança. DataGramaZero - Revista de Ciência da Informação, Rio de Janeiro, v. 8, n. 1, fev. 2007. Disponível em: <http://www.dgz.org.br/fev07/Art_04.htm>. Acesso em: 4 jun. 2013.

NASSAR, Paulo. História e cultura organizacional. Revista Comunicação Empresarial, São Paulo, amo 10, n. 36, 2000.

OLIVEIRA, Djalma de Pinho Rebouças. Sistemas de informações gerenciais: estratégicas, táticas e operacionais. 10. ed. São Paulo: Atlas, 2004.

RESENDE, Denis Alcides; ABREU, Aline França de. Tecnologia da informação aplicada a sistemas de informação empresariais. São Paulo: Atlas. 2006.

SCHEIN, Edgar H. Cultura organizacional e liderança. São Paulo: Atlas. 2009.

TARAPANOFF, Kira. Inteligência, informação e conhecimento. In: MCLNERNEY, Claire R. Compartilhamento e gestão do conhecimento: profissionais da informação em um ambiente de confiança mútua. Brasília: IBICT, 2006. Cap. 3, p. 57-72.

WERSIG, Gernot; NEVELING, Ultrich. The phenomena of interesting to information science. Information Scientist, London, v. 9. n. 4, p. 127140, Dec. 1975. 
WILSON, Thomas D. Information management. In: FEATHER, John; STURGES, Paul. INTERNATIONAL ENCYCLOPEDIA OF INFORMATION AND LIBRARY SCIENCE. London: Routledge, 1997. p. 187-196.

\section{Title}

The relationship between information, organizational culture and decision making in an organization

\section{Abstract}

Introduction: Includes a documentary research on the relationship between information, organizational culture and decision making in an organization.

Objective: The goal is to check the influence of information, considering the organizational culture, decision making in an organization.

Methodology: The literature review include authors specialized in the areas of information (Belkin; Borko; Capurro; Choo; Tarapanoff; among others); culture (Fleury et al.; Moraes and Fadel; Nassar and Schein), decision making (Angeloni; Hoppen; Leitão and Nassif; Lousada and Valentim and Oliveira) and organization (Bernardes and Marcondes and Maximiano).

Results: That there may be a strong interdependency between information, culture and decision making in an organization.

Conclusions: The information can facilitate understanding of the culture of an organization, how the processes of change occur and what alternatives can be raised so that she can achieve success in their decision-making process in order to ensure its perpetuation over time.

Keywords: Information organization. Culture in the organizational environment. Decision making in organization. Information in the decision-making process.

\section{Titulo}

La relación entre la informacion, cultura organizacional y toma de decisiones en una organización

\section{Resumen}

Introducción: Incluye de una investigación documental sobre la relación entre la información, la cultura organizacional y la toma de decisiones en una organización.

Objetivo: Es comprobar la influencia de la información, teniendo en cuenta la cultura de la organización, toma de decisiones en una organización decisión.

Metodología: La revisión de la literatura incluye autores especializados en las áreas de información (Belkin; Borko; Capurro; Choo; Tarapanoff; entre otros), la cultura (Fleury et al.; Moraes y Fadel; Nassar y Schein), la toma de 
Paulo Danelon Lopes; Valmira Perucchi

A relação entre informação, cultura organizacional e tomada de decisão em uma organização

decisiones (Angeloni; Hoppen; Leitão y Nassif; Lousada y Valentim y Oliveira) y organización (Marcondes y Bernardes y Maximiano).

Resultados: Puede haber una fuerte interdependencia entre la información, la cultura y la toma de decisiones en una organización.

Conclusiones: La información puede facilitar la comprensión de la cultura de una organización, cómo se producen los procesos de cambio y qué alternativas se pueden plantear para que pueda alcanzar el éxito en su proceso de toma de decisiones con el fin de garantizar su perpetuación en el tiempo.

Palabras clave: Información para la organización. Cultura en el entorno de la organización. La toma de decisiones en la organización. iNformación en el proceso de toma de decisiones.

Recebido em: 11/12/2014

Aceito em: 01/07/2015 